\title{
Coherent Backscattering of Bose-Einstein Condensates in Two-Dimensional Disorder Potentials
}

\author{
Michael Hartung, ${ }^{1}$ Thomas Wellens, ${ }^{2}$ Cord A. Müller, ${ }^{3}$ Klaus Richter, ${ }^{1}$ and Peter Schlagheck ${ }^{1}$ \\ ${ }^{1}$ Institut für Theoretische Physik, Universität Regensburg, 93040 Regensburg, Germany \\ ${ }^{2}$ Physikalisches Institut, Albert-Ludwigs Universität Freiburg, 79104 Freiburg, Germany \\ ${ }^{3}$ Physikalisches Institut, Universität Bayreuth, 95440 Bayreuth, Germany \\ (Received 23 April 2008; revised manuscript received 14 June 2008; published 11 July 2008)
}

\begin{abstract}
We study quantum transport of an interacting Bose-Einstein condensate in a two-dimensional disorder potential. In the limit of a vanishing atom-atom interaction, a sharp cone in the angle-resolved density of the scattered matter wave is observed, arising from constructive interference between amplitudes propagating along reversed scattering paths. Weak interaction transforms this coherent backscattering peak into a pronounced dip, indicating destructive instead of constructive interference. We reproduce this result, obtained from the numerical integration of the Gross-Pitaevskii equation, by a diagrammatic theory of weak localization in the presence of nonlinearity.
\end{abstract}

The past years have witnessed an increasing number of theoretical and experimental research activities on the behavior of ultracold atoms in magnetic or optical disorder potentials [1-15]. A central aim in this context is the realization and unambiguous identification of strong Anderson localization with Bose-Einstein condensates, which was attempted by several experimental groups [1$3]$ with recent success $[4,5]$, and theoretically studied both from the perspective of the expansion process of the condensate $[6,7]$ as well as from the scattering perspective $[8,9]$. Complementary studies were focused on localization properties of Bogoliubov quasiparticles [10,11], on dipole oscillations in the presence of disorder [12,13], as well as on the realization of Bose glass phases $[14,15]$.

The above-mentioned topics (apart from Ref. [7]) mainly refer to processes that are essentially one dimensional (1D) by nature. Qualitatively new phenomena, however, do arise in two or three spatial dimensions, due to the scenario of weak localization. The latter manifests in a slight reduction of the transmission probability of an incident wave through a disordered region as compared to the classically expected value, due to constructive interference between backscattered paths and their time-reversed counterparts. This interference phenomenon particularly leads to a cone-shaped enhancement of the backscattering current in the direction reverse to the incident beam, which was indeed observed [16] and theoretically analyzed [17] in light scattering processes from disordered media. Related weak localization effects also arise in electronic mesoscopic physics, leading to characteristic peaks in the magnetoresistance $[18,19]$.

In this Letter, we investigate the phenomenon of coherent backscattering with atomic Bose-Einstein condensates that propagate in presence of two-dimensional (2D) disorder potentials. An essential ingredient that comes into play here is the interaction between the atoms of the condensate. On the mean-field level, this is accounted for by the nonlinear term in the Gross-Pitaevskii equation describing the time evolution of the condensate wave function. Indeed, nonlinearities do also appear in scattering processes of light, e.g., from a gas of cold atoms, due to the saturation of the intra-atomic transition [20-22]. In this case, however, the saturation also leads to inelastic scattering [21,22] and, in addition, the nonlinearity competes with other dephasing mechanisms induced, e.g., by polarization phenomena [23] or thermal motion [24]. The complementary process of atomic condensates scattering from optical random potentials in the mean-field regime provides a cleaner situation where the coherence of the atomic wave function remains well preserved in the presence of the nonlinearity. As we shall argue below, this leads to substantial modifications of the coherent backscattering feature. In particular, the interaction turns constructive into destructive interference, leading to a negative coherent backscattering peak height. This is reminiscent of the weak antilocalization effects due to spin-orbit interaction observed for mesoscopic magnetotransport [25].

The starting point of our investigation is the timedependent 2D Gross-Pitaevskii equation describing the mean-field dynamics of the condensate in presence of the disorder potential $V(\vec{r})[\vec{r} \equiv(x, y)]$,

$$
\begin{aligned}
i \hbar \frac{\partial}{\partial t} \psi(\vec{r}, t)= & \left(-\frac{\hbar^{2}}{2 m} \Delta+V(\vec{r})+\tilde{g}(x)|\psi(\vec{r}, t)|^{2}\right) \psi(\vec{r}, t) \\
& +S(t) \delta\left(x-x_{0}\right) \exp (-i \mu t / \hbar)
\end{aligned}
$$

where $\psi(\vec{r}, t)$ is the condensate wave function and $S(t)$ denotes a source term simulating the coherent injection of matter waves with chemical potential $\mu$ from an external reservoir onto the scattering region [8]. In the numerical integration of Eq. (1), $S(t)$ is adiabatically increased from zero to a final value $S_{0}$ that corresponds to a fixed incident current density $j_{\text {in }}$. Periodic boundary conditions are imposed on the transverse boundaries (in $y$ direction) of the numerical grid to ensure a homogeneous flow in absence of disorder, whereas absorbing boundary conditions 
applied at the edges of the longitudinal ( $x$ ) direction allow us to inhibit artificial backreflection of outgoing waves with rather high accuracy [26].

In Eq. (1), the effective 2D interaction strength is written as $\tilde{g}(x) \equiv \hbar^{2} g(x) /(2 m)$, with the dimensionless nonlinearity parameter $g(x)$. In the presence of a harmonic confinement of the condensate in the third spatial dimension with the oscillator length $a_{\perp}(x) \equiv \sqrt{\hbar /\left[m \omega_{\perp}(x)\right]}$, we have $g(x)=4 \sqrt{2 \pi} a_{s} / a_{\perp}(x)$, where $a_{s}$ denotes the $s$-wave scattering length of the atoms. We assume that $g(x)$ is adiabatically ramped on and off in front of and behind the disorder region, as shown in Fig. 1. This spatial variation of the nonlinearity would correspond to a finite extent of the transverse harmonic confinement into which the condensate is propagating. As for the disorder potential $V(\vec{r})$, we choose a Gaussian random process characterized by a vanishing mean value $\langle V(\vec{r})\rangle=0$ and a Gaussian correlation function $\langle V(\vec{r}) V(\vec{r}+\Delta \vec{r})\rangle=V_{0}^{2} e^{-\Delta r^{2} / 2 \sigma^{2}}$ with correlation length $\sigma$. We focus in the following on the parameters $k \sigma=0.5$, with $k \equiv \sqrt{2 m \mu} / \hbar$ the wave number of the incident beam, and $V_{0} / \mu=0.614$. The incident current density reads $j_{\text {in }}=\hbar k\left|\psi_{0}\right|^{2} / m$, where we set $\psi_{0}=$ $k$ for the incident amplitude [27].

At the above values for $\sigma$ and $V_{0}$, scattering in the disorder region is approximately isotropic. This is quantitatively expressed by the equivalence of the two relevant length scales that the disorder averages introduces for the transport process of the condensate: the scattering mean free path $\ell_{s}$, which describes the average decay of the
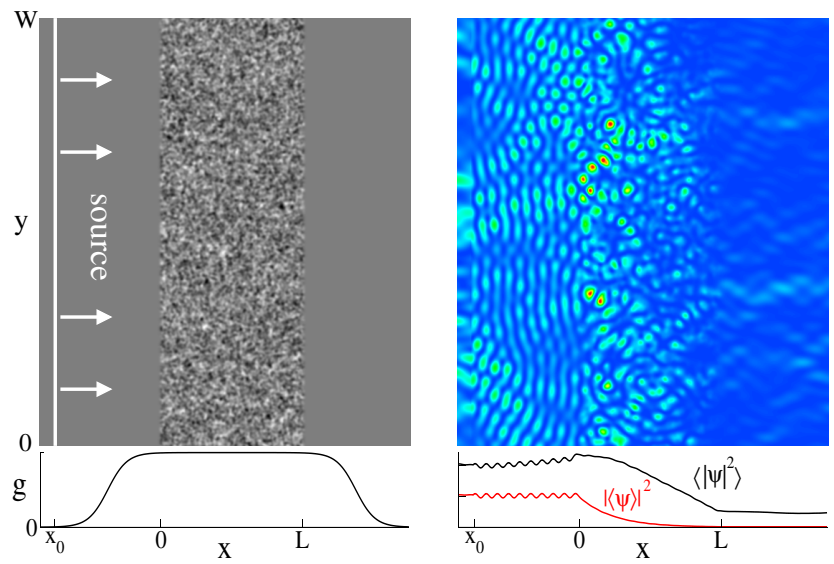

FIG. 1 (color online). Scattering geometry and stationary scattering state associated with a randomly generated disorder potential. The left-hand side displays $V(x, y)$ in a gray-scale plot and shows the spatial variation of the nonlinearity $g(x)$. The upper right panel shows the density of the corresponding scattering state that is populated through the numerical integration of the inhomogeneous Gross-Pitaevskii equation (1). The lower right panel shows the decay of the coherent mode $|\langle\psi\rangle|^{2}$ and the density $\left\langle|\psi|^{2}\right\rangle$ with $x$, averaged over $y$ for $\sim 10^{3}$ randomly generated disorder configurations. Parameters: $k L=40, k W=$ $120, k \sigma=0.5, V_{0}=0.614 \mu, g=0.005, j_{\text {in }}=\hbar k^{3} / m$, with $k \equiv \sqrt{2 m \mu} / \hbar$. incident coherent mode inside the disorder region according to $|\langle\psi(\vec{r})\rangle|^{2} \propto \exp \left(-x / \ell_{s}\right)$, and the transport mean free path $\ell_{\mathrm{tr}}$, which characterizes the decay of the average density $\left\langle|\psi(\vec{r})|^{2}\right\rangle$ (see Fig. 1). In the absence of nonlinearity, the scattering mean free path is in leading order in $V_{0}$ given by the Born approximation

$$
\left(k \ell_{s}\right)^{-1} \simeq(\pi / 2)\left(V_{0} / \mu\right)^{2}(k \sigma)^{2} I_{0}\left(k^{2} \sigma^{2}\right) \exp \left(-k^{2} \sigma^{2}\right),
$$

where $I_{j}(\xi)$ is the modified Bessel function of order $j$.

The transport mean free path can be extracted from the linear decrease of $\left\langle|\psi(\vec{r})|^{2}\right\rangle$ with $x$ according to $\left\langle|\psi(\vec{r})|^{2}\right\rangle \propto$ $\left(L+z_{0} \ell_{\mathrm{tr}}-x\right)$, with $z_{0}=0.82$ in two spatial dimensions, and $L$ the longitudinal extent of the disorder region. In lowest order in $V_{0}, \ell_{\mathrm{tr}}$ is given by the Boltzmann transport mean free path $\ell_{B}$ defined through

$$
\ell_{s} / \ell_{B}=1-I_{1}\left(k^{2} \sigma^{2}\right) / I_{0}\left(k^{2} \sigma^{2}\right) .
$$

Weak localization effects lead to logarithmic corrections that yield for $k \ell_{B} \gg 1[28,29]$

$$
\ell_{\mathrm{tr}} \simeq \ell_{B}\left[1-2\left(k \ell_{B}\right)^{-1} \log \left(L / \ell_{B}\right)\right] \text {. }
$$

As shown in Fig. 2, the expressions (2) and (4) are in good agreement with the numerically computed values of $\ell_{s}$ and $\ell_{\text {tr }}$ for $V_{0}<\mu$. Specifically at $k \sigma=0.5$ and $V_{0} / \mu=$ 0.614 , we find $k \ell_{s} \simeq 9.61$ and $k \ell_{\text {tr }} \simeq 9.75$.

The angle-resolved current in backward direction is numerically computed from the decomposition of the reflected wave $\psi_{\text {ref }}(x, y) \equiv \psi(x, y)-\psi_{0} \exp (i k x)$ at fixed position $x$ close to $x_{0}$ [where $g(x)$ is negligibly small] into the transverse eigenmodes $\chi_{n}(y) \sim \exp ($ in $\pi y / W)$, which support outgoing waves into the directions with the angles $\theta_{n} \equiv \arcsin [2 \pi n /(k W)]$. Figure 3 shows the average angular density $j(\theta)$ of the backscattered current, which is normalized such that $\int_{0}^{2 \pi} j(\theta) d \theta=2 \pi$. In the linear case $(g=0)$, we encounter the well-known cone structure at $\theta=0$, which is a characteristic signature of weak localization [16,17]. Rather small values of $g \sim 0.02$ corresponding to $\tilde{g}|\psi(\vec{r})|^{2} \sim 10^{-2} \mu$, are sufficient to substantially modify this cone-shaped peak. Most interestingly, it is not washed out by the nonlinearity, but

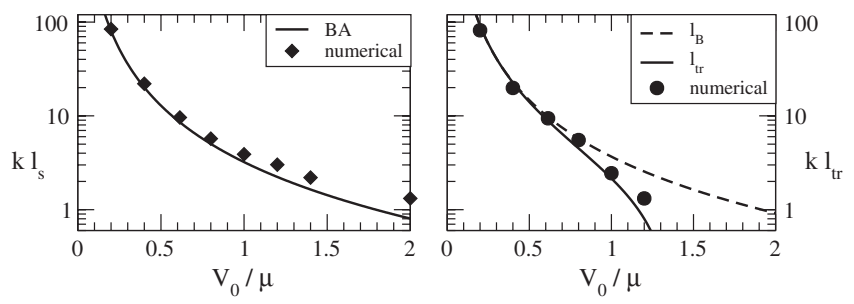

FIG. 2. Scattering mean free path $\ell_{s}$ (left panel) and transport mean free path $\ell_{\text {tr }}$ (right panel) in the disorder potential for $k \sigma=$ 0.5 in the absence of nonlinearity. In the left panel, the numerically computed lengths are compared with the Born approximation (2) (solid line) and in the right panel with the Boltzmann mean free path (3) $\left(\ell_{B}\right.$, dashed line) and the expression (4) that takes into account weak localization corrections $\left(\ell_{\mathrm{tr}}\right.$, solid line). We find $\ell_{\mathrm{tr}} \simeq \ell_{s}$ for $V_{0}<\mu$. 


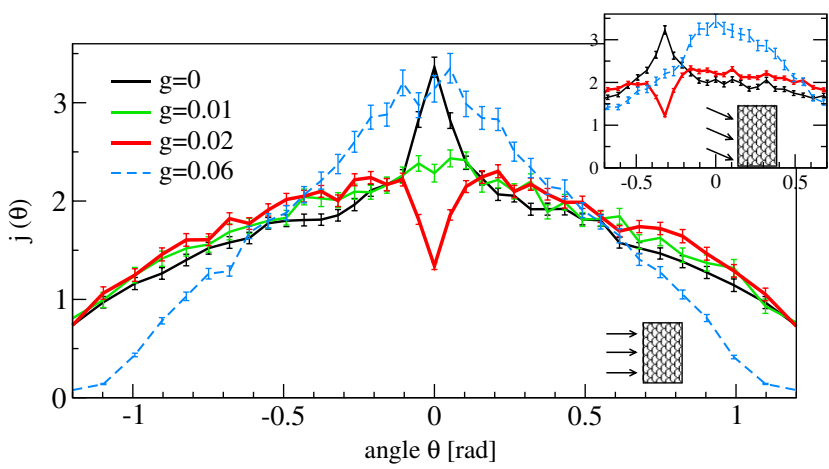

FIG. 3 (color online). Angle-resolved current density of backscattered atoms in the absence and presence of nonlinearity, obtained from the average over $\sim 10^{3}$ disorder configurations (parameters as in Fig. 1; the error bars denote the statistical standard deviation). The coherent backscattering cone for $g=0$ (black line) is transformed into a pronounced dip for intermediate nonlinearities ( $g=0.02$, bold red line), and turns into a smooth peak structure at larger values of $g(g=0.06$, dashed blue line). The inset shows the angle-resolved current for the case of a tilted incident beam where the source term in Eq. (1) populates the transverse eigenmode $\chi_{n=6}(y)$ defined by the angle $\theta_{6} \simeq 0.32$. In contrast to the smooth peak, the cone and dip structures are indeed found at the angle that corresponds to retroreflection of the incident beam, which confirms that they both arise due to interference between reflected paths.

transformed into a dip that roughly has the same shape as the peak at $g=0$. This indicates that the underlying interference phenomenon between reflected scattering paths is still effective at finite $g$, but has turned from constructive to destructive.

The occurrence of a dip in the backscattered current is confirmed by calculations based on the diagrammatic approach for weak localization in presence of a nonlinearity [22,30]. Assuming the realization of a stationary scattering state, the average density $\left\langle|\psi(\vec{r})|^{2}\right\rangle$ is expressed in terms of ladder diagrams, which amounts to neglecting interference, and thus describing wave transport as a classical random walk. This assumption is valid approximately for a dilute medium, i.e., for $k \ell \gg 1$ with $\ell \equiv \ell_{B} \simeq \ell_{\mathrm{tr}} \simeq \ell_{s}$ (for isotropic scattering). Furthermore, we assume the condition $g^{2}\left|\psi_{0} / k\right|^{4} k \ell \ll 1$ under which scattering from the fluctuations $\tilde{g}|\psi(\vec{r})|^{2}$ of the nonlinear refractive index is negligible compared to scattering from the disorder potential $V(\vec{r})$ [31]. Therefore, the average density $\left\langle|\psi(\vec{r})|^{2}\right\rangle$ remains approximately unaffected by the nonlinearity, and thus is well described by linear transport theory. From the average density, the flux backscattered in direction $\theta=0$ results as $j_{L}(0)=\int_{0}^{L} d x \exp (-x / \ell) \times$ $\left\langle|\psi(x)|^{2}\right\rangle /\left(\ell\left|\psi_{0}\right|^{2}\right)$.

In a second step, the coherent backscattering peak is calculated by means of crossed (Cooperon) diagrams, describing interference between reversed scattering paths. Following the diagrammatic approach presented in Ref. [30], we obtain the height of the coherent backscattering peak from the transport equations

$$
\begin{aligned}
C_{c}(x)= & \left|\psi_{0}\right|^{2} e^{-\hat{x} / \ell}\left(1+\frac{i}{k} \int_{x_{0}}^{x} d x^{\prime} g\left(x^{\prime}\right) C_{1}\left(x^{\prime}\right)\right), \\
C_{1}(x)= & \int_{0}^{L} \frac{d x^{\prime}}{\pi \ell}\left[K_{0}\left(\left|\frac{\hat{x}-x^{\prime}}{\ell}\right|\right)\left(C_{1}\left(x^{\prime}\right)+C_{c}\left(x^{\prime}\right)\right)\right. \\
& +\frac{i}{k} K_{1}\left(\left|\frac{\hat{x}-x^{\prime}}{\ell}\right|\right)\left\langle\left|\psi\left(x^{\prime}\right)\right|^{2}\right\rangle \\
& \left.\times \int_{\min \left(x, x^{\prime}\right)}^{\max \left(x, x^{\prime}\right)} d x^{\prime \prime} g\left(x^{\prime \prime}\right)\left(C_{1}\left(x^{\prime \prime}\right)+C_{c}\left(x^{\prime \prime}\right)\right)\right]
\end{aligned}
$$

for the "Cooperon intensity" $C_{1}(x)$ and the "coherent Cooperon intensity" $C_{c}(x)$, with $\hat{x} \equiv \max (x, 0)$ and $K_{0,1}$ the modified Bessel functions of the second kind. The contribution to the flux scattered in backward direction then results as

$$
\begin{aligned}
j_{C}(0)= & \operatorname{Re} \int_{0}^{L} \frac{d x}{\ell\left|\psi_{0}\right|^{2}} e^{-x / \ell}\left(C_{1}(x)+\frac{i}{k}\left\langle|\psi(x)|^{2}\right\rangle\right. \\
& \left.\times \int_{x_{0}}^{x} d x^{\prime} g\left(x^{\prime}\right) C_{1}\left(x^{\prime}\right)\right) .
\end{aligned}
$$

Note that nonlinear processes also occur for $x_{0}<x<0$ where $V(\mathbf{r})=0$ but $g(x)>0$ (see Fig. 1). Hence, the cone height $j_{C}(0)$-in contrast to the background intensity $j_{L}(0)$ - explicitly depends on the spatial extent of the nonlinearity region in front of the disorder potential, and can therefore be tuned through the ramp-up of $g(x)$.

In the absence of nonlinearity, the above equations reduce to linear transport theory in a two-dimensional slab, yielding $\left.C_{1}(x)\right|_{g=0}=\left\langle|\psi(x)|^{2}\right\rangle-\left|\psi_{0}\right|^{2} \exp (-x / \ell)$. We then obtain $\left.j_{C}(0)\right|_{g=0}=j_{L}(0)-1 / 2$, which expresses reciprocity symmetry, i.e., the equality of reversed path amplitudes (the term $1 / 2$ describes single scattering). For $g \neq 0$, however, the nonlinearity turns $C_{1}(x)$ into a complex quantity, as evident from the terms proportional to $i g$ in Eqs. (5)-(7). This indicates an effective phase difference between the reversed scattering paths. Consequently, the backscattered current $j_{C}(0)$ is expected to decrease with increasing nonlinearity, and may even become negative if this phase difference is sufficiently large.

This latter situation is indeed encountered if the set of Eqs. (5)-(7) is numerically solved for the system parameters under consideration. As shown in Fig. 4, the total flux $j_{L}(0)+j_{C}(0)$ resulting from Eqs. (5)-(7) (solid line) agrees rather well with the average value for $j(0)$ obtained from the numerical simulation (symbols). While we can identify the relevant diagrams that give rise to the dip, we have no straightforward explanation of this phenomenon in simple physical terms. It appears, however, fundamentally different from interaction-induced dephasing in electronic transport processes through disordered systems [32,33], which require finite temperatures and do not allow for a description in terms of a single coherent wave due to the fermionic nature of the particles.

At larger nonlinearities $g \geq 0.03$ the numerical propagation of the inhomogeneous Gross-Pitaevskii equation (1) 


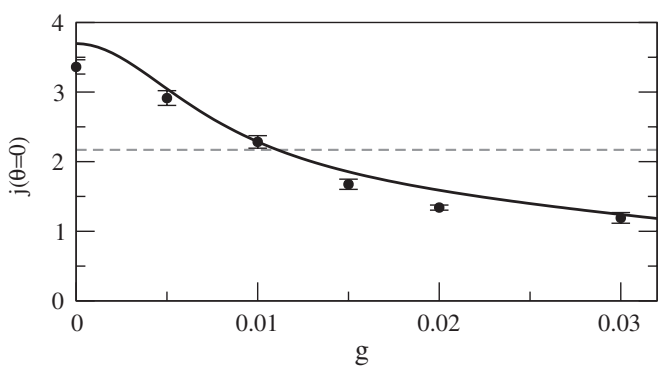

FIG. 4. Backscattered current at $\theta=0$ as a function of the nonlinearity $g$ (parameters as in Fig. 1), obtained from the numerical simulation (symbols) and from the diagrammatic theory, Eqs. (5)-(7) (solid line). The horizontal dashed line indicates the diffuse background intensity $j_{L}(0)$. Negative cone heights $j_{C}(0)<0$ leading to a dip in the angle-resolved current density $j(\theta)$ appear for $g>0.01$.

does not converge to a stationary scattering state, but leads to a permanently time-dependent behavior of $\psi(\vec{r}, t)$, as predicted in Refs. [31] and encountered also in the transport of condensates through 1D disorder potentials [8]. In this regime, the average backscattered current again displays a peak around $\theta=0$; this peak is, however, comparatively broad and does not arise from a coherent backscattering phenomenon. This becomes obvious if we inject the incident wave with a finite angle onto the disorder region. While the cone and dip structures at $g=0$ and 0.02 appear, as shown in the inset of Fig. 3, at the expected angle of coherent backscattering, corresponding to retroreflection of the incident beam, the broad peak at $g=0.06$ is not affected in this way.

In conclusion, the presence of a small nonlinearity reverts the scenario of weak localization and gives rise to a cone-shaped dip, instead of a peak, in the angle-resolved backscattered current density. This phenomenon appears to be rather robust; it is numerically encountered also for disorder potentials with longer correlation lengths $\sigma$ giving rise to anisotropic scattering, and we expect its manifestation also in three spatial dimensions (as predicted by the diagrammatic theory) as well as for speckle disorder where diagrammatic approaches would have to be based on the treatment of Ref. [29]. We therefore believe that the effect would be measurable, for a reasonably large range of parameters, in state-of-the-art transport experiments with coherent Bose-Einstein condensates in well-controlled disorder potentials.

We thank A. Buchleitner, D. Delande, B. Grémaud, R. Kuhn, and T. Paul for inspiring discussions. Funding through DFG (Forschergruppe 760) and Bayerisches Eliteförderungsgesetz is gratefully acknowledged.

[1] D. Clément et al., Phys. Rev. Lett. 95, 170409 (2005).
[2] C. Fort et al., Phys. Rev. Lett. 95, 170410 (2005).

[3] T. Schulte et al., Phys. Rev. Lett. 95, 170411 (2005).

[4] J. Billy et al., Nature (London) 453, 891 (2008).

[5] G. Roati et al., Nature (London) 453, 895 (2008).

[6] L. Sanchez-Palencia et al., Phys. Rev. Lett. 98, 210401 (2007).

[7] S.E. Skipetrov, A. Minguzzi, B. A. van Tiggelen, and B. Shapiro, Phys. Rev. Lett. 100, 165301 (2008).

[8] T. Paul, P. Leboeuf, N. Pavloff, K. Richter, and P. Schlagheck, Phys. Rev. A 72, 063621 (2005).

[9] T. Paul, P. Schlagheck, P. Leboeuf, and N. Pavloff, Phys. Rev. Lett. 98, 210602 (2007).

[10] N. Bilas and N. Pavloff, Eur. Phys. J. D 40, 387 (2006).

[11] P. Lugan et al., Phys. Rev. Lett. 98, 170403 (2007).

[12] J.E. Lye et al., Phys. Rev. A 75, 061603(R) (2007).

[13] Y.P. Chen et al., Phys. Rev. A 77, 033632 (2008).

[14] B. Damski, J. Zakrzewski, L. Santos, P. Zoller, and M. Lewenstein, Phys. Rev. Lett. 91, 080403 (2003).

[15] L. Fallani, J. E. Lye, V. Guarrera, C. Fort, and M. Inguscio, Phys. Rev. Lett. 98, 130404 (2007).

[16] M. P. Van Albada and A. Lagendijk, Phys. Rev. Lett. 55, 2692 (1985); P.-E. Wolf and G. Maret, Phys. Rev. Lett. 55, 2696 (1985).

[17] E. Akkermans, P.E. Wolf, and R. Maynard, Phys. Rev. Lett. 56, 1471 (1986).

[18] B. L. Altshuler, D. Khmel'nitzkii, A. I. Larkin, and P. A. Lee, Phys. Rev. B 22, 5142 (1980).

[19] G. Bergmann, Phys. Rep. 107, 1 (1984).

[20] T. Chanelière, D. Wilkowski, Y. Bidel, R. Kaiser, and C. Miniatura, Phys. Rev. E 70, 036602 (2004).

[21] V. Shatokhin, C. A. Müller, and A. Buchleitner, Phys. Rev. Lett. 94, 043603 (2005).

[22] T. Wellens, B. Grémaud, D. Delande, and C. Miniatura, Phys. Rev. A 73, 013802 (2006).

[23] T. Jonckheere, C. A. Müller, R. Kaiser, C. Miniatura, and D. Delande, Phys. Rev. Lett. 85, 4269 (2000).

[24] G. Labeyrie, D. Delande, R. Kaiser, and C. Miniatura, Phys. Rev. Lett. 97, 013004 (2006).

[25] D. M. Zumbühl, J. B. Miller, C. M. Marcus, K. Campman, and A. C. Gossard, Phys. Rev. Lett. 89, 276803 (2002).

[26] T. Shibata, Phys. Rev. B 43, 6760 (1991).

[27] A different choice of $\psi_{0}$ would amount to rescaling $g$.

[28] L.P. Gor'kov, A.I. Larkin, and D.E. Khmel'nitskiı̌, Pis'ma Zh. Eksp. Teor. Fiz. 30, 248 (1979) [JETP Lett. 30, 228 (1979).

[29] R. C. Kuhn, C. Miniatura, D. Delande, O. Sigwarth, and C. A. Müller, Phys. Rev. Lett. 95, 250403 (2005); R. C. Kuhn, O. Sigwarth, C. Miniatura, D. Delande, and C. A. Müller, New J. Phys. 9, 161 (2007).

[30] T. Wellens and B. Grémaud, Phys. Rev. Lett. 100, 033902 (2008).

[31] B. Spivak and A. Zyuzin, Phys. Rev. Lett. 84, 1970 (2000); J. Opt. Soc. Am. B 21, 177 (2004); S.E. Skipetrov and R. Maynard, Phys. Rev. Lett. 85, 736 (2000).

[32] B. L. Altshuler and A. G. Aronov, in Electron-electron Interaction in Disordered Systems, edited by A.L. Efros and M. Pollak (Elsevier, Amsterdam, 1985), p. 1.

[33] G. Zala, B. N. Narozhny, and I. L. Aleiner, Phys. Rev. B 64, 214204 (2001). 